\title{
Comunidades sintéticas arbóreas: una alternativa al deterioro forestal en la parte baja de la cuenca del río Usumacinta en Tabasco, México
}

\author{
Synthetic arboreal communities: an alternative to forest deterioration \\ in the lower part of the Usumacinta river basin in Tabasco, Mexico
}

\section{Xicoténcatl de la Cruz-Uc a , Arturo Valdés-Manzanilla a*, Víctor L Barradas ${ }^{\text {b, }}$ Luisa del Carmen Cámara Cabrales ${ }^{\text {a }}$}

\begin{abstract}
*Autor de correspondencia: ${ }^{a}$ Universidad Juárez Autónoma de Tabasco, División Académica de Ciencias Biológicas, Carretera Villahermosa-Cárdenas km 05, Villahermosa, Tabasco, México, tel.: +52-993-3544308, arturo.valdes@ujat.mx

b Universidad Nacional Autónoma de México, Instituto de Ecología, México, D.F., México, vbarrada@ecologia.unam.mx
\end{abstract}

\section{SUMMARY}

\begin{abstract}
A new methodology is presented to design synthetic arboreal communities, with emphasis on forest management in deforested regions. This methodology is based on the potential distribution of native tree species of Mexico in the lower part of the Usumacinta river basin in southeastern Mexico. The potential distributions of native species were obtained from the probability of occurrence calculated by the Maxent ecological niche model, using presence data, from an area delimited by the municipalities of Balancán and Tenosique, Tabasco, Mexico, which has had a strong deforestation in the last decades. A synthetic arboreal community is proposed for this study area, which consists of the five species that have the largest areas of potential distribution, according to the calculations made with a geographic information system, which covers a considerable area of the study region $\left(1,951.9 \mathrm{~km}^{2}\right)$, maximizing the reforested area. Two of the species studied are listed within some type of national or international standard on threatened species.
\end{abstract}

Key words: modeling, potential distribution, native species.

\section{RESUMEN}

Se presenta una nueva metodología para diseñar comunidades sintéticas arbóreas con énfasis en manejo forestal en regiones deforestadas. Dicha metodología está basada en la distribución potencial de especies arbóreas nativas de México en la parte baja de la cuenca del río Usumacinta en el sureste de México. Las distribuciones potenciales de las especies nativas se obtuvieron de la probabilidad de ocurrencia calculada por el modelo de nicho ecológico Maxent, usando datos de presencia, de una zona delimitada por los municipios de Balancán y Tenosique, Tabasco, México, que ha tenido una fuerte deforestación en las últimas décadas. Se propone una comunidad sintética arbórea para esta zona de estudio que consta de las cinco especies que tienen las mayores áreas de distribución potencial, que abarca un área de 1.951,9 km² de la región de estudio, maximizando el área reforestada. Dos de las especies estudiadas están listadas dentro de algún tipo de norma nacional o internacional sobre especies amenazadas.

Palabras clave: modelado, distribución potencial, especies nativas.

\section{INTRODUCCIÓN}

La deforestación que conlleva el cambio de uso de suelos a nivel mundial es un problema notorio y cada vez más grave a la par del supuesto desarrollo económico. Los bosques ocupan actualmente unos 4.000 millones de hectáreas, que representa cerca del $31 \%$ de la superficie del planeta (World bank 2017). Las mayores tasas de deforestación se registran en bosques de la zona templada situados en América del Norte, Asia y Europa. Sin embargo, desde principios del siglo veinte en la zona templada han disminuido el ritmo de deforestación, aumentando en los bosques tropicales (FAO 2016).
En México existe un acelerado proceso de deforestación de bosques y selvas, ya que de las aproximadamente $18 \times 10^{6}$ hectáreas de selvas, para el año 2002 solo quedaban $3.15 \times 10^{6}$ hectáreas que representan el $17,5 \%$ de la vegetación original, transformando el uso de suelo forestal a agrícola y pecuario, convirtiéndose en grandes extensiones de pastizales, creando parches de bosque templado y tropical dando una gran fragmentación (Tudela 1992, Moreno et al. 2011, Vázquez et al. 2011). En Tabasco, se estima que existían aproximadamente $1.5 \times 10^{6}$ hectáreas de selva mediana y alta perennifolia antes de 1900 . Mucha de esa superficie ahora está convertida en pastizales (Tudela 1990). Los municipios de Balancán y Tenosique también 
presentan dicha degradación ambiental por causa de la deforestación, donde la mitad de la superficie selvática se ha convertido en praderas, donde antes existía vegetación de selva mediana de pukte (Bucida buceras L.) y selva baja de tinto (Haematoxylum campechianum) (Cámara et al. 2011), con abundancia de árboles maderables, medicinales y de otros tipos; mientras que otra superficie importante de esta región, se incursiona actualmente en nuevos usos de suelo, como cultivos anuales, plantaciones maderables (Cedrella odorata L.) y no maderables como palma de aceite (Alaeis guinnensis Jacq.) y frutales, entre otros (Márquez et al. 2008, Vázquez et al. 2011).

Los estudios referentes a la distribución potencial de especies son abundantes en plantas y animales usando diversos métodos, tales como Maxent o GARP, para lograr tal fin. Por ejemplo, De Cauwer et al. (2014), utilizando el modelo de nicho Maxent, concluyen que la distribucion realizada y potencial de Pterocarpus angolensis DC, en el sur de África son muy similares, siendo una especie muy amenazada por la sobreexplotación, debido a su madera valiosa, y por los cambios de uso del suelo.

En Italia, en un estudio sobre la probabilidad de ocurrencia de Quercus suber L., Vessella y Schirone (2013), usando entre otros métodos de ocurrencia al modelo Maxent, encuentran similitudes en las predicciones entre Maxent y el modelo GARP para la modelación del nicho ecológico de dicha especie. Otro estudio llevado a cabo en las estribaciones del Himalaya, India, con Justicia adhato$d a$ L., demuestra que esta especie tiene una gran distribución potencial en esa región (Yang et al. 2013).

En el ecosistema costero conocido como Bosque Atlántico en Brasil, con fines de conservación, se estiman la distribución potencial de cinco especies de Metzgeria, destacándose que el $93 \%$ de los fragmentos de bosque podrían ser protegidos (Barros et al. 2012). En Ecuador se analiza la distribución geográfica, conservación y estado poblacional de Grosvenoria campii King et Rob. (Asteraceae), generando modelos de distribución potencial, que indican que la especie no es rara sino poco recolectada (Caranqui y Suárez-Duque 2012). En México hay un estudio sobre la distribución potencial de Taxus globosa Schlecht., especie de conífera con distribución restringida y listada bajo estatus de conservación, encontrándose que las principales comunidades vegetales donde potencialmente se puede distribuir esta especie son los bosque de pino-encino, de encino-pino, de pino y mesófilo de montaña, en los estados de Hidalgo, Nuevo León, Puebla, Tamaulipas y Veracruz (García-Aranda et al. 2012).

Una comunidad sintética arbórea es definida en este estudio como aquella que surge de la interacción de dos o más especies arbóreas que convergen en un área de estudio en común, con la potencialidad y posibilidad de coexistir en un nicho realizado. De igual manera, esta comunidad debe de enfocarse a los fines específicos que se pretendan impulsar en una cierta región, ya sea de mejorar la conservación y aumentar la biodiversidad o de opti- mizar el manejo forestal. Diversos estudios se han hecho sobre comunidades arbóreas como el realizado en Costa Rica, donde se proponen sistemas cacao-maderables como una alternativa para satisfacer las necesidades de subsistencia de los agricultores, obteniendo ingresos adicionales y mejorando al mismo tiempo la capacidad del agroecosistema para proporcionar otros servicios ambientales, por lo que sugieren para tal fin, el uso de Cordia alliodora, por ser un árbol de rápido crecimiento y con buena calidad de madera para la fabricación de muebles, además de que se regenera naturalmente en sitios despejados (Somarriba et al. 2014). Este árbol se encuentra asociado con pastizales, mientras que las zonas fragmentadas están dominadas por árboles como Tabebuia rosea (Griscom y Ashton 2010).

Sprenger et al. (2013) sugieren que la plantación de mezclas de pioneros y especies de árboles tolerantes a la sombra lleva a índices de filtración de agua al suelo moderados, ya que las alturas de los árboles y la apertura del dosel, están compensadas en comparación con los monocultivos de cualquiera de las especies arbóreas de crecimiento rápido o lento. También ellos sugieren que los árboles afectan a las aguas subterráneas, a través de la evaporación, absorción de agua por la raíz y la interceptación de la lluvia de acuerdo a su altura, la apertura del dosel y la biomasa.

El presente estudio tiene como finalidad diseñar comunidades sintéticas arbóreas, a partir de la distribución potencial de especies nativas de la parte baja de la cuenca del río Usumacinta, delimitada para los municipios de Balancán y Tenosique en Tabasco, México. Esta región es considerada una zona altamente deforestada, lo que permitirá a los tomadores de decisiones elegir algunas comunidades (o solo algunas de las especies para construir sus propias comunidades) para diversificar las plantaciones existentes, y a los conservacionistas les proporcionará una herramienta útil para conocer las especies que pueden coexistir en una misma zona con el fin de enriquecer el hábitat o conocer los lugares más propicios para la reforestación y así enfocar los recursos de una manera más eficiente. La relevancia del estudio es que representa una de las primeras aproximaciones para construir comunidades sintéticas obtenidas de la distribución potencial de especies arbóreas nativas en la región de estudio, generadas a partir de un modelo de nicho de distribución potencial de especies (Maxent) y con énfasis hacia el diseño de comunidades sintéticas arbóreas que abarquen la mayor área posible de la región de estudio, para reforestarla con especies que puedan servir para diversos usos como maderables o de mejoramiento de la biodiversidad.

\section{MÉTODOS}

Perspectiva del área de estudio. Para el presente estudio, en el estado de Tabasco, la región de la cuenca del río Usumacinta se delimitó para los municipios de Balancán y Tenosique (figura 1). La superficie total del área de estudio correspondió a $3.732,8 \mathrm{~km}^{2}$. En la zona se presentaron tres 

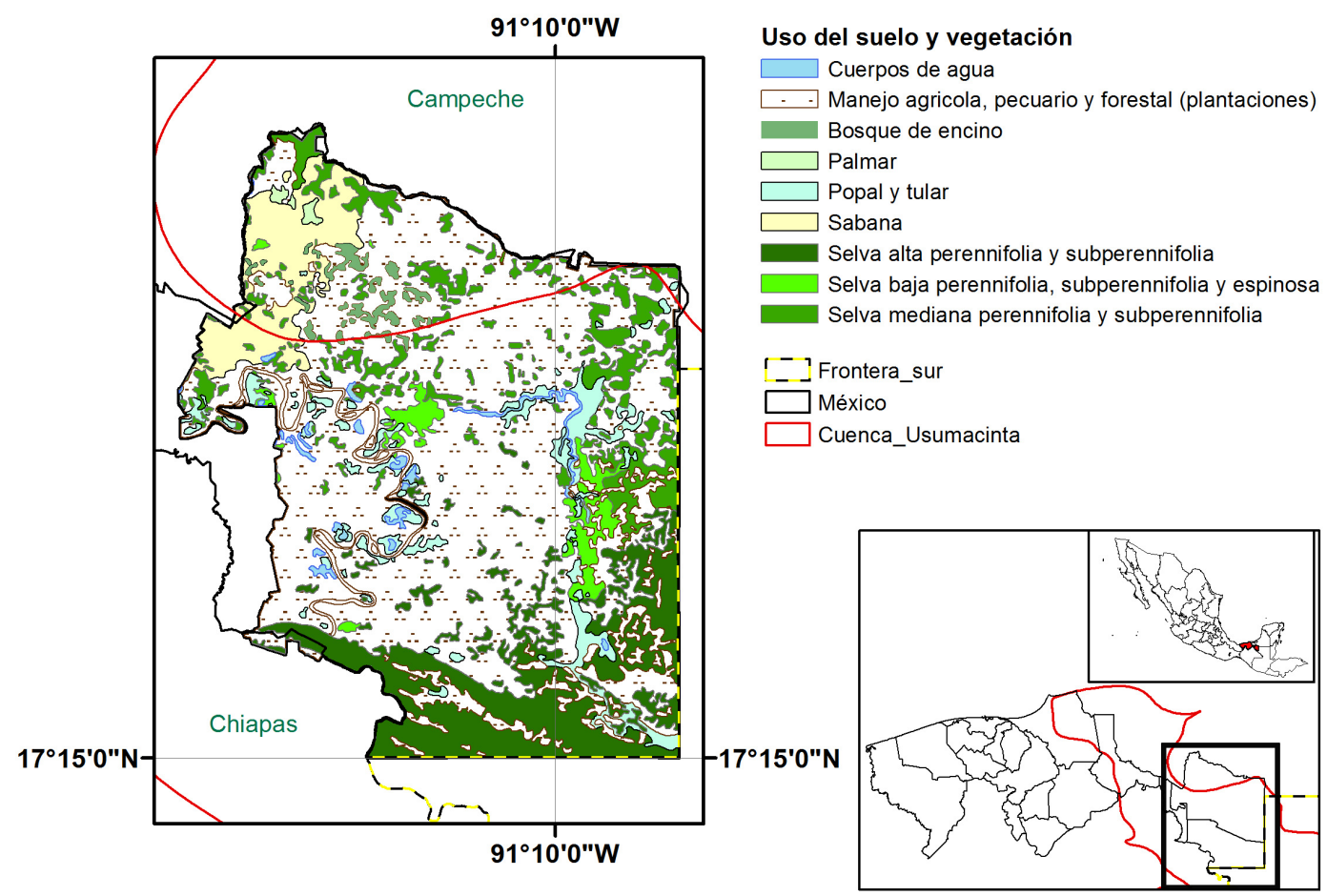

Figura 1. Uso del suelo y distribución de la vegetación en el área de estudio ubicada en la cuenca del río Usumacinta en México.

Land use and vegetation distribution in the study area located on the Usumacinta river basin in Mexico.

tipos de clima, según la clasificación de Köppen: clima cálido húmedo con abundantes lluvias en verano $(\mathrm{Am})$ con régimen térmico medio anual fluctuando de $24^{\circ} \mathrm{C}$ a $28^{\circ} \mathrm{C}$; clima cálido húmedo con lluvias todo el año (Af) con la temperatura media anual oscilando de $22{ }^{\circ} \mathrm{C}$ a $28^{\circ} \mathrm{C}$ y clima tipo cálido subhúmedo con lluvias en verano (Aw) con temperatura media anual mayor de $26^{\circ} \mathrm{C}$. La precipitación total anual para Balancán fue de 1.500 a $2.000 \mathrm{~mm}$ y para Tenosique de 2.000 a $2.500 \mathrm{~mm}$.

Se encontraron los suelos leptosoles, gleysoles, luvisoles y plintosoles, pero el tipo de suelo dominante fue el vertisol éutrico. El uso del suelo se dividió en pastizales (66\% de la superficie), vegetación secundaria (12,5\%), vegetación hidrófita $(9,9 \%)$, selvas medianas y bajas con $3,7 \%$ y otros usos con 7,9\% (lagunas, poblaciones, agricultura, ganadería).

Entre 1970 y 1980 la ganadería sustituyó las últimas zonas selváticas que se encontraban dispersas principalmente en los municipios de Balancán y Tenosique. En dicha zona, el territorio se ordenó de acuerdo con un plan de sustitución de las selvas por pastizales implementado por el gobierno federal mexicano llamado Plan BalancánTenosique, sin tener en cuenta el impacto en los recursos naturales (Manjarrez et al. 2007, Cámara et al. 2011).

Selección de especies. Se seleccionaron 15 especies arbóreas nativas de la región tropical mexicana (cuadro 1) y que tenían diversos usos, sobre todo maderables, por lo cual podían ser de interés comercial (Pennington y Sarukhán 2005). Dicha selección fue motivada por el interés de repoblar una zona altamente deforestada del sureste mexicano donde anteriormente la selva era el ecosistema predominante (Tudela 1992). De cada especie se colectó información biológica, incluyendo dispersión y longevidad de las semillas, el tipo de crecimiento, función que cumple durante la sucesión, así como la dureza de la madera y si se encontraban en alguna categoría de riesgo o amenazada de acuerdo a la Norma Oficial Mexicana NOM059-SEMARNAT-2010, de la Unión Internacional para la Conservación de la Naturaleza (UICN) y de la Convención sobre el Comercio Internacional de Especies Amenazadas de Fauna y Flora Silvestres (CITES).

Para la selección de las 15 especies elegidas se utilizaron datos obtenidos en campo, del herbario de la División Académica de Ciencias Biológicas (DACBiol) de la Universidad Juárez Autónoma de Tabasco (UJAT), de Pennington y Sarukhán (2005) y de la búsqueda realizada en las siguientes bases de datos: REMIB de la Comisión Nacional para el conocimiento y uso de la Biodiversidad (CONABIO 2015) y GBIF de la Global Biodiversity Information Facility (GBIF 2015).

Base de datos y distribución potencial. Para la búsqueda de los registros de ocurrencia de las 15 especies elegidas se usó la base de datos GBIF, con los filtros de búsqueda de nombre científico de las especies, país (México) y del 
periodo de 1960 a 2010. Se realizó un control de calidad de los datos de ocurrencias donde se descartaron las coordenadas, datos duplicados y se corroboraron los nombres científicos de las especies elegidas.

Las capas ambientales utilizadas fueron las de edafología, regiones biológicas, uso de suelo y vegetación, precipitación anual, temperatura media anual, temperatura mínima promedio, temperatura máxima promedio, evapotranspiración anual para todo México, que se obtuvieron de CONABIO, tenían un tipo categórico y una resolución espacial de 0,017 grados decimales en coordenadas geográficas con un datum WGS84. Las capas que correspondían a la cuenca del río Usumacinta se obtuvieron de GeoWebRum del Centro GEO y del Centro del Cambio Global y la Sustentabilidad en el Sureste (CCGSS) y tenían las mismas características de las capas ambientales.

Se eligió el modelo de nicho Maxent versión 3.3.3e por ser un software de libre acceso, su facilidad para utilizarse en la obtención de distribuciones potenciales con pocos registros de ocurrencias de especies y ser uno de los mejores métodos de modelación de distribución de especies (Phi1lips et al. 2006, Caranqui y Suárez-Duque 2012, GarcíaAranda et al. 2012). El software se corrió con la mayoría de sus parámetros default como 10.000 puntos de background, un máximo de 500 iteraciones, un porcentaje de $25 \%$ de puntos elegidos en forma aleatoria como datos de prueba, pero sin las características "threshold" y "hinge" para evitar que se sobre-ajustaran las curvas de respuesta.

Para evaluar el desempeño del Maxent en el cálculo de las distribuciones potenciales, se usaron los parámetros Area Under the Curve (AUC; área bajo la curva) y True Skill Statistics (TSS) (Allouche et al. 2006).

Para evitar predictores correlacionados se siguió la metodología de Van Gils et al. (2014), donde se dividieron los predictores en dos tipos: climáticos y no climáticos, que se iban eliminando de acuerdo al porcentaje de la contribución de cada variable al modelo, usando el procedimiento jacknife y los valores de AUC y TSS. Para ello se corrió varias veces el software, hasta encontrar el número mínimo de variables poco correlacionadas de cada tipo $(<6)$, que mantuvieran un valor alto de AUC y TSS.

El software de manejo de Sistemas de Información Geográfica (GIS) ArcMap 10.1 se usó para modificar la distribución potencial de cada especie estimada por Maxent para adaptarla a la usada en este estudio. El formato raster de salida generado en Maxent para cada especie, se exportó al GIS. Primeramente, con el GIS se calculó el porcentaje de la probabilidad de presencia de la distribución obtenida en Maxent, dividiéndose los porcentajes de hábitat en cinco categorías: muy bajo $(<25 \%)$, bajo $(25-$ $42 \%$ ), medio (43-59\%), alto (60-74\%) y muy alto (75$100 \%)$. Posteriormente, se generó una nueva distribución potencial modificada para cada especie, donde se unieron las áreas correspondientes a las categorías medio, alto y muy alto, mediante el comando geoprocessing dissolve del GIS en un archivo con formato shapefile. Posteriormente, con estos nuevos mapas (uno por especie), se generaron las comunidades sintéticas, donde se calculó el área de intersección entre las distribuciones potenciales modificadas de varias especies, mediante el comando geoprocessing, intersect del GIS. Finalmente, se combinaron las especies de mayor a menor área de distribución potencial modificada con el criterio de maximizar el área deforestada, donde se excluyeron las especies que tenían un área de intersección menor al $10 \%$ del área de estudio. El proceso metodológico se muestra en la figura 2.

\section{RESULTADOS}

Los cálculos para encontrar la distribución potencial de cada especie dieron una AUC promedio de 0,950 con una desviación estándar de 0,028 y un TSS promedio de 0,723 con una desviación estándar de 0,088 , lo que indicaba un buen desempeño de Maxent para determinar la distribución potencial. El número total de ocurrencias de especies usadas para los cálculos fue de 11.977, con una media de 798 y una desviación de 726, encontrándose un máximo de 3.210 ocurrencias para Bursera simaruba y un mínimo de 189 para Blepharidium mexicanum.

Todas las especies tuvieron una distribución potencial total que abarcó toda la zona de estudio (cuadro 1). Sin embargo, una vez descartadas las probabilidades de ocurrencia bajas y muy bajas $(<42 \%), B$. simaruba fue la que tuvo la mayor distribución potencial resultante o área de intersección (cuadro 1). Por otro lado, las especies con menor área de intersección fueron Simarouba glauca, Metopium brownei, Lysiloma latisiliquum y Cordia dodecandra. La especie Astronium graveolens se encontraba en categoría A (amenazada) de la norma mexicana NOM-059-SEMARNAT-201. En el mismo contexto, la especie $C$. alliodora se encontraba en el status LC de la UICN (cuadro 1).

El énfasis en este estudio ha sido reforestar la mayor área posible del área de estudio, en detrimento de la biodiversidad, por lo que se escogió B. simaruba como especie base para la combinación con otras especies, ya que es la especie que posee la mayor distribución potencial resultante o área de intersección en la región de estudio (figura $3 \mathrm{~A}$ ), ya que abarcó casi toda el área de estudio (cuadro 1), aún sin su área con probabilidad de ocurrencia muy baja y baja o sea menor que el $42 \%$ (figura $3 \mathrm{~B}$ ). Solo se usaron ocho especies para diseñar diferentes comunidades sintéticas, porque las siete especies restantes no se pudieron combinar o presentaron un área de intersección muy pequeña ( $<10 \%$ del área de estudio), tal es el caso de las especies S. glauca, M. brownei, L. latisiliquum y $C$. dodecandra (cuadro 1). Para hacer las combinaciones de especies incluidas en las comunidades sintéticas, se tomó en cuenta el tamaño del área de intersección de cada especie (cuadro 1), en forma descendente, empezando con B. Simaruba, la de mayor superficie, y terminando con $A$. graveolens, ya que H. campechianum, que era la octava en área de intersección, no se pudo combinar con las demás especies. El área 


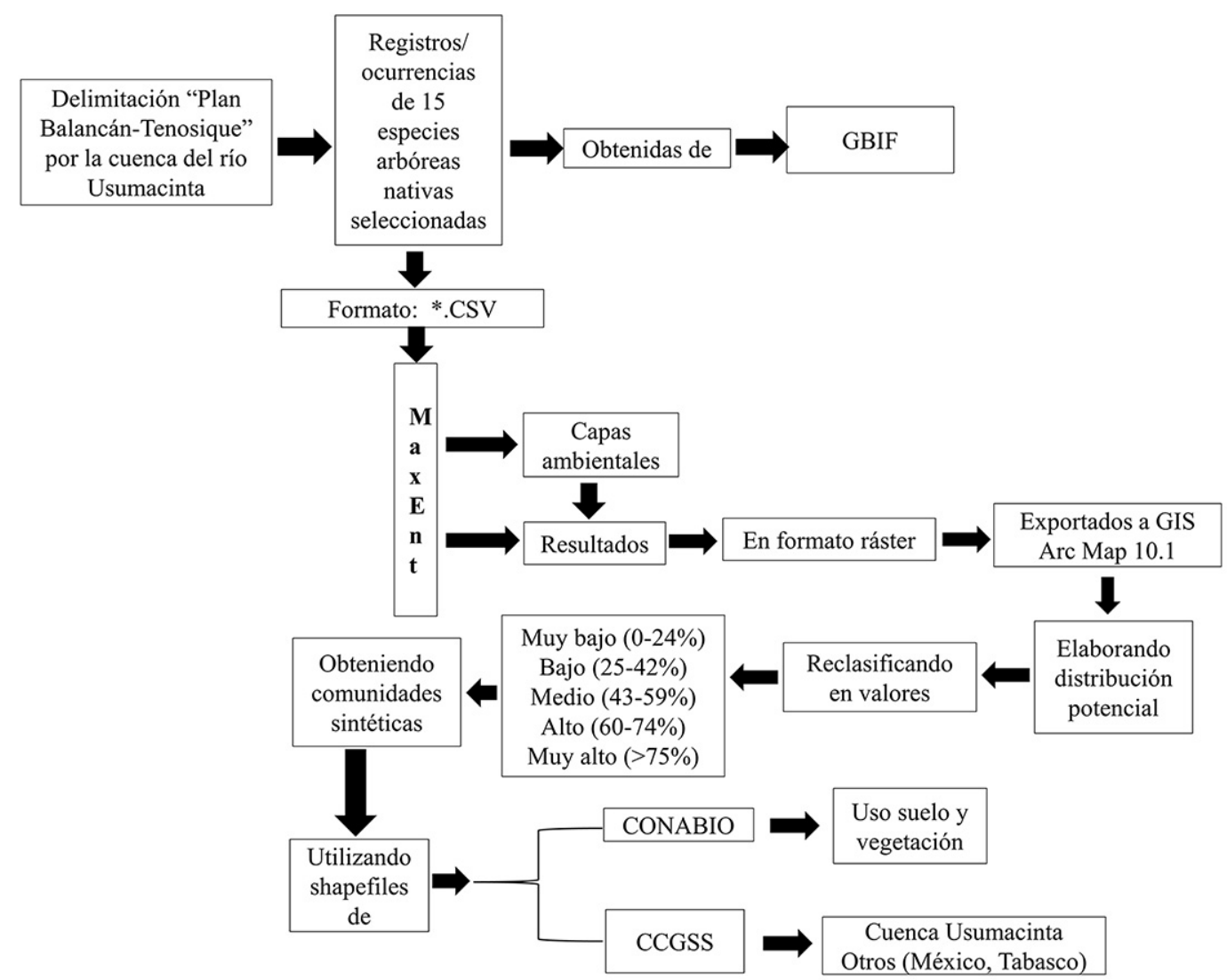

Figura 2. Diagrama metodológico para la obtención de las comunidades sintéticas arbóreas, donde CONABIO significa Comisión Nacional para el conocimiento y Uso de la Biodiversidad, DACBiol significa División Académica de Ciencias Biológicas, GBIF es la Global Biodiversity Information Facility y CCGSS significa Centro de Cambio Global y la Sustentabilidad en el Sureste.

Methodological diagram for obtaining the synthetic arboreal communities, where CONABIO means National Commission for the knowledge and Usage of Diversity, DACBiol means Academic Division of Biologic Sciences, GBIF is the Global Biodiversity Information Facility and CCGSS means Center of Global Change and Sustainability in the Southeast.

de intersección o de congruencia varió desde el $88 \%$ en una combinación de dos especies, hasta el $3 \%$ en una combinación de 8 especies (cuadro 2), lo que indicó que entre mayor fuera el número de especies combinadas, era menor el área de intersección o congruencia entre ellas, esto se puede apreciar en la figura 4A que muestra una comunidad sintética de cinco especies que tuvo un área de intersección o congruencia mucho más grande que la de la figura $4 \mathrm{~B}$ donde se muestra una comunidad sintética de solo ocho especies, lo que recalcó la importancia de enfatizar hacia que fines se enfocaría la comunidad sintética diseñada, ya sea al manejo forestal, donde se buscaría maximizar el área reforestada, o a la conservación y aumento de la biodiversidad, que requeriría una superficie mucho menor de la zona de estudio.

\section{DISCUSIÓN}

Ha habido varios intentos en diferentes partes del mundo para diseñar comunidades sintéticas arbóreas enfocadas a ciertos fines como conservación, reforestación, biodiver- sidad, tal es el caso de la comunidad cacao-árboles maderables sugerida por Somarriba et al. (2014) en Costa Rica. Sin embargo, faltan nuevos métodos con mayor objetividad para diseñar estas comunidades. Este estudio trata de ayudar a mejorar esa carencia, a través de la propuesta de una nueva metodología para diseñar comunidades sintéticas arbóreas enfocadas a reforestar una cierta región.

En cuanto a la elección de las especies nativas utilizadas en este estudio, el criterio para elegirlas es que sea factible establecer plantaciones con estas especies y además que estas tengan diversos usos y beneficios económicos para los pobladores de la zona de estudio. Cámara et al. (2011) sugieren establecer plantaciones de $C$. pentandra, T. rosea, L. latisiliquum, C. alliodora, P. ellipticum, B. mexicanum, B. simaruba, $C$. dodecandra y $H$. campechianum en esta región de estudio, lo que corrobora la selección de las especies nativas escogidas, ya que algunas de ellas están incluidas tanto en la comunidad sintética de cinco especies (figura 4A), como en la de ocho especies (figura 4B). 
Cuadro 1. Especies nativas escogidas, AUC significa área bajo la curva, TSS significa estadístico de habilidad verdadera y Área de intersección es el área de la distribución potencial de una especie sin las probabilidades de ocurrencia bajas y muy bajas, donde $P$ es la probabilidad de ocurrencia aleatoria.

Chosen native species, AUC means area under the curve, TSS means true skill statistics and Intersection Area is the area of potential distribution of a species without the low and very low occurrence probabilities, where $P$ is the random occurrence probability.

\begin{tabular}{|c|c|c|c|c|c|c|}
\hline Nombre científico & $\begin{array}{l}\text { Nombre } \\
\text { común }\end{array}$ & $\begin{array}{c}\text { AUC } \\
P=0,5\end{array}$ & TSS & $\begin{array}{c}\text { Distribución } \\
\text { potencial }\left(\mathrm{km}^{2}\right)\end{array}$ & $\begin{array}{c}\text { Área de } \\
\text { intersección }\left(\mathrm{km}^{2}\right)\end{array}$ & $\begin{array}{l}\text { Norma de } \\
\text { protección }\end{array}$ \\
\hline Bursera simaruba (L.) Sarg. & Palo mulato & 0,895 & 0,678 & $3.721,6$ & $3.516,4$ & \\
\hline Spondias mombin L. & Jobo & 0,927 & 0,639 & $3.723,4$ & $3.441,3$ & \\
\hline Blepharidium mexicanum Standl. & Popiste & 0,985 & 0,831 & $3.721,2$ & $3.276,1$ & \\
\hline Tabebuia rosea (Bertol.) DC. & Macuilis & 0,928 & 0,707 & $3.723,4$ & $2.839,6$ & \\
\hline Cordia alliodora (Ruiz et Pav.) Oken & Bojón & 0,938 & 0,727 & $3.725,1$ & $2.615,5$ & $\begin{array}{l}\text { Least concern LC } \\
\text { (preocupación } \\
\text { menor) Lista roja de } \\
\text { la UICN de especies } \\
\text { amenazadas }\end{array}$ \\
\hline Ceiba pentandra (L.) Gaertn. & Ceiba & 0,949 & 0,640 & $3.723,4$ & $2.168,1$ & \\
\hline $\begin{array}{l}\text { Pseudobombax ellipticum (Kunth) } \\
\text { Dugand }\end{array}$ & Amapola & 0,900 & 0,532 & $3.723,4$ & $1.771,3$ & \\
\hline Haematoxylum campechianum L. & Tinto & 0,969 & 0,750 & $3.723,4$ & $1.440,9$ & \\
\hline Astronium graveolens Jacq. & Jobillo & 0,948 & 0,730 & $3.723,4$ & 985,6 & $\begin{array}{c}\text { A (amenazada) } \\
\text { NOM-059- } \\
\text { SEMARNAT-2010 }\end{array}$ \\
\hline Piscidia pisipula (L.) Sarg. & Jabín & 0,949 & 0,735 & $3.699,8$ & 697,4 & \\
\hline Simarouba glauca DC. & Zapatero & 0,969 & 0,637 & $3.710,4$ & 208,5 & \\
\hline $\begin{array}{l}\text { Swartzia cubensis (Britton et Wilson) } \\
\text { Standl. }\end{array}$ & $\begin{array}{l}\text { Corazón } \\
\text { azul }\end{array}$ & 0,984 & 0,821 & $3.708,9$ & 120,6 & \\
\hline Metopium brownei (Jacq.) Urb. & Chechen & 0,975 & 0,768 & $3.722,5$ & 21,4 & \\
\hline Lysiloma latisiliquum (L.) Benth. & Tzalam & 0,976 & 0,866 & $3.708,9$ & 6,2 & \\
\hline Cordia dodecandra DC. & Siricote & 0,966 & 0,792 & $3.718,9$ & 0 & \\
\hline
\end{tabular}

Cuadro 2. Áreas obtenidas a partir de la distribución potencial y de la formación de comunidades sintéticas usando las especies nativas escogidas.

Obtained areas from the potential distribution and formation of synthetic communities using the selected native species.

\begin{tabular}{|c|c|c|c|}
\hline Comunidad sintética & $\begin{array}{l}\text { Número de } \\
\text { especies }\end{array}$ & $\begin{array}{c}\text { Superficie } \\
\left(\mathrm{km}^{2}\right)\end{array}$ & $\begin{array}{c}\text { \% total del área } \\
\left(3.732,8 \mathrm{~km}^{2}\right)\end{array}$ \\
\hline B. simaruba, S. mombin. & 2 & $3.277,7$ & 87,8 \\
\hline B. simaruba, S. mombin, B. mexicanum. & 3 & $2.903,0$ & 77,8 \\
\hline B. simaruba, S. mombin, B. mexicanum, T. rosea. & 4 & $2.244,7$ & 60,1 \\
\hline B. simaruba, $S$. mombin, B. mexicanum, T. rosea, C. alliodora. & 5 & $1.951,9$ & 52,3 \\
\hline $\begin{array}{l}\text { B. simaruba, S. mombin, B. mexicanum, T. rosea, C. alliodora, C. } \\
\text { pentandra. }\end{array}$ & 6 & $1.358,9$ & 36,4 \\
\hline $\begin{array}{l}\text { B. simaruba, S. mombin, B. mexicanum, T. rosea, C. alliodora, } C \text {. } \\
\text { pentandra, P. ellipticum. }\end{array}$ & 7 & 714,0 & 19,1 \\
\hline $\begin{array}{l}\text { B. simaruba, S. mombin, B. mexicanum, T. rosea, C. alliodora, } C \text {. } \\
\text { pentandra, P. ellipticum, A. graveolens. }\end{array}$ & 8 & 116,5 & 3,1 \\
\hline
\end{tabular}



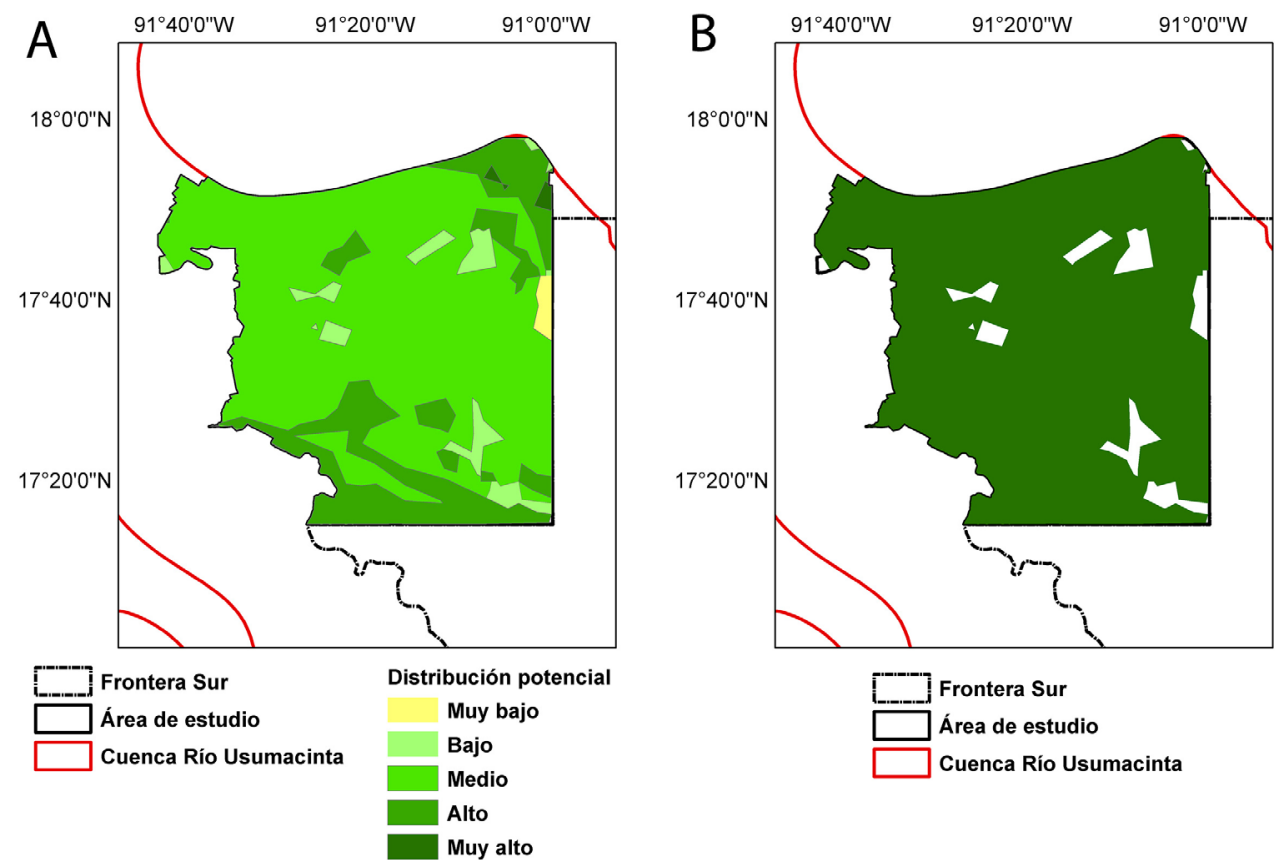

Figura 3. A) Mapa de la distribución potencial de B. Simaruba para diferentes categorias de porcentajes de habitat adecuado de ocurrencia (bajo, medio, alto, muy alto). B) Lo mismo que A), pero sin el área de las categorías de porcentaje muy bajo y bajo de habitat adecuado.

Map of the potential distribution of Bursera simaruba for different categories of percentages of suitable habitat (low, medium, high, very high). B) The same as A), but without the area of category of very low and low percentage of suitable habitat.
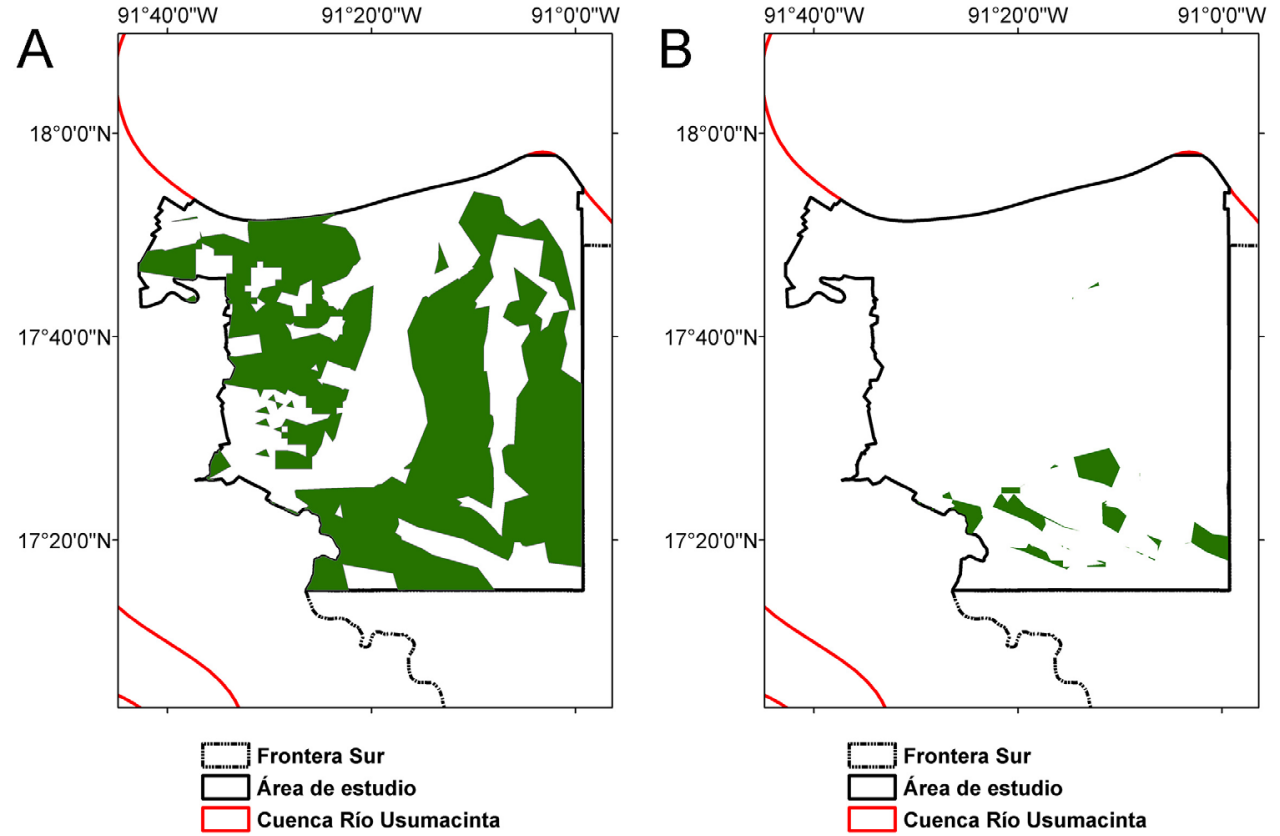

Figura 4. A) Comunidad sintética arbórea que consta de cinco especies: B. simaruba, S. mombin, B. mexicanum, T. rosea y C. Alliodora. B) Comunidad sintética arbórea que excluye especies con distribución potencial restringida y que consta de ocho especies: B. simaruba, S. mombin, B. mexicanum, T. rosea, C. Alliodora, C. pentranda, P. ellipticum y A. graveolens.

A) Synthetic arboreal community consisting of five species: Bursera simaruba, Spondias mombin, Blepharidium mexicanum, Tabebuia rosea and Cordia alliodora. B) Synthetic arboreal community excluding species with restricted potential distribution and consisting of eight species: B. simaruba, S. mombin, B. mexicanum, T. rosea, C. alliodora, Ceiba pentandra, Pseudobombax ellipticum and Astronium graveolens. 
Otras características de las especies influyeron en su inclusión en este estudio, por ejemplo A. graveolens es pionera en la sucesión (fijando nitrógeno) y es considerada como una madera tropical dura usada en la construcción de casas (cuadro 3) según Griscom y Ashton (2010); C. pentandra es una especie que aparece a finales de la sucesión (Griscom y Ashton 2010); C. alliodora también es pionera y de larga duración (Griscom y Ashton 2010) y enlistada en la categoría LC de la UICN (UICN 2017); Tabebuia $s p$. es de larga vida y pionera, siendo todas estas especies con dispersión de semilla anemócora (cuadro 3). Por otro lado, B. simaruba y S. mombin son pioneras y su síndrome de dispersión es zoocora (cuadro 3), siendo estas especies anteriormente mencionadas de rápido crecimiento y maderables (Griscom y Ashton 2010).
Sautu et al. (2006) mencionan otras características como la longevidad de la conservación de las semillas de $C$. pentandra (2 meses), C. alliodora ( $<1 \mathrm{mes}), S$. mombin (30 meses) y $T$. rosea (11 meses), destacando la superioridad de $S$. mombin en la germinación, lo que permite que las semillas de estas especies se puedan conservar un mayor tiempo, para germinarse y sembrarse cuando sea necesario.

En cuanto a las comunidades sintéticas, dado el énfasis en este estudio hacia la reforestación de la mayor área posible de la región de estudio, se piensa que la comunidad sintética formada por cinco especies (figura 4A) podría ser la más adecuada, ya que esta mejor balanceada que la otra comunidad de ocho especies (figura 4B) en los siguientes aspectos:

Cuadro 3. Principales características biológico-forestales de las especies utilizadas en el presente estudio. Fuente: Pennington y Sarukhán (2005).

Main biological-forest characteristics of used species in the present study. Source: Pennington and Sarukhán (2005).

\begin{tabular}{|c|c|c|c|c|c|c|c|c|}
\hline Especie & $\begin{array}{c}\text { Tolerancia a } \\
\text { la sombra }\end{array}$ & Crecimiento & $\begin{array}{c}\text { Monoico / } \\
\text { Dioico }\end{array}$ & Floración & Polinización & $\begin{array}{l}\text { Dispersión de } \\
\text { semillas }\end{array}$ & $\begin{array}{c}\text { Preferencia } \\
\text { de suelo }\end{array}$ & Madera \\
\hline T. rosea & $\begin{array}{l}\text { Intolerante- } \\
\text { intermedio }\end{array}$ & $\begin{array}{l}\text { Lento a } \\
\text { moderado }\end{array}$ & Monoecius & Enero-mayo & Abejas & Anemócora & Calizos & $\begin{array}{l}\text { Para } \\
\text { muebles }\end{array}$ \\
\hline S. mombin & Intolerante & $\begin{array}{l}\text { Moderado } \\
\text { rápido }\end{array}$ & Dioecius & Marzo-junio & $\begin{array}{l}\text { Mariposas, } \\
\text { abejas }\end{array}$ & Zoócora & $\begin{array}{l}\text { Arenosos/ } \\
\text { arcillosos }\end{array}$ & $\begin{array}{l}\text { Madera } \\
\text { suave }\end{array}$ \\
\hline B. mexicanum & $\begin{array}{c}\text { Intermedia a } \\
\text { tolerante }\end{array}$ & $\begin{array}{l}\text { Lento a } \\
\text { moderado }\end{array}$ & Monoecius & $\begin{array}{l}\text { Julio- } \\
\text { diciembre }\end{array}$ & Aves & Zoócora & Calcáreos & $\begin{array}{l}\text { Madera } \\
\text { dura }\end{array}$ \\
\hline B. simaruba & Intolerante & $\begin{array}{l}\text { Moderado a } \\
\text { rápido }\end{array}$ & $\begin{array}{l}\text { Monoecius } \\
\text { o Dioecius }\end{array}$ & $\begin{array}{l}\text { Febrero- } \\
\text { agosto }\end{array}$ & Abejas & Zoócora & Calcáreos & $\begin{array}{l}\text { Madera } \\
\text { suave }\end{array}$ \\
\hline C. pentandra & Intolerante & Rápido & Monoecius & $\begin{array}{l}\text { Diciembre- } \\
\text { marzo }\end{array}$ & Murciélagos & Viento y agua & $\begin{array}{l}\text { Arenosos o } \\
\text { arcillosos }\end{array}$ & $\begin{array}{l}\text { Madera } \\
\text { blanda }\end{array}$ \\
\hline S. glauca & Intolerante & $\begin{array}{l}\text { Moderado } \\
\text {-rápido }\end{array}$ & Dioecius & $\begin{array}{l}\text { Febbrero- } \\
\text { abril }\end{array}$ & Polillas & Zoócora & Calizos & Blanda \\
\hline $\begin{array}{l}H . \\
\text { campechianum }\end{array}$ & Intolerante & $\begin{array}{l}\text { Moderado a } \\
\text { rápido }\end{array}$ & Monoecius & $\begin{array}{l}\text { Septiembre- } \\
\text { abril }\end{array}$ & Abejas & Anemócora & $\begin{array}{l}\text { Arcillosos } \\
\text { inundables }\end{array}$ & $\begin{array}{l}\text { Colo- } \\
\text { rantes }\end{array}$ \\
\hline P. pisipula & Intolerante & $\begin{array}{l}\text { Moderado } \\
\text {-rápido }\end{array}$ & Monoecius & Mayo-julio & Abejas & Anemócora & Calizos & Dura \\
\hline C. alliodora & Intolerante & $\begin{array}{l}\text { Moderado } \\
\text {-rápido }\end{array}$ & Monoecius & Agosto-abril & Entomófila & Anemócora & Arcillosos & Buena \\
\hline P. ellipticum & Intolerante & $\begin{array}{l}\text { Moderado a } \\
\text { rápido }\end{array}$ & Monoecius & Enero-junio & $\begin{array}{l}\text { Murciélagos } \\
\text { y aves }\end{array}$ & Anemócora & $\begin{array}{l}\text { Profundos } \\
\text { y calizos }\end{array}$ & $\begin{array}{l}\text { Madera } \\
\text { blanda }\end{array}$ \\
\hline A. graveolens & Tolerante & Intermedio & Dioecius & $\begin{array}{l}\text { Marzo- } \\
\text { mayo }\end{array}$ & Abejas & Anemócora & $\begin{array}{l}\text { Calizos y } \\
\text { aluviales }\end{array}$ & $\begin{array}{l}\text { Constru- } \\
\text { cción }\end{array}$ \\
\hline C. dodecandra & $\begin{array}{l}\text { Intolerante- } \\
\text { intermedio }\end{array}$ & $\begin{array}{l}\text { Lento- } \\
\text { moderado }\end{array}$ & Monoecius & $\begin{array}{l}\text { Octubre- } \\
\text { enero }\end{array}$ & $\begin{array}{l}\text { Colibríes y } \\
\text { murciélagos }\end{array}$ & Zoócora & $\begin{array}{l}\text { Pobres y } \\
\text { someros }\end{array}$ & $\begin{array}{l}\text { Para } \\
\text { muebles }\end{array}$ \\
\hline S. cubensis & Intolerante & $\begin{array}{l}\text { Lento- } \\
\text { moderado }\end{array}$ & Monoecius & $\begin{array}{l}\text { Febrero- } \\
\text { mayo }\end{array}$ & Abejas & Zoócora & Calizos & Dura \\
\hline L. latisiliquum & Intolerante & $\begin{array}{l}\text { Moderado- } \\
\text { rápido }\end{array}$ & Monoecius & Marzo-junio & Abejas & $\begin{array}{l}\text { Lanzamiento } \\
\text { del fruto }\end{array}$ & $\begin{array}{l}\text { Calcáreos y } \\
\text { someros }\end{array}$ & $\begin{array}{l}\text { Madera } \\
\text { dura }\end{array}$ \\
\hline M. brownei & Intolerante & $\begin{array}{l}\text { Moderado a } \\
\text { rápido }\end{array}$ & Monoecius & $\begin{array}{l}\text { Marzo- } \\
\text { mayo }\end{array}$ & Abejas & $\begin{array}{l}\text { Zoócora } \\
\text { (Aves) }\end{array}$ & $\begin{array}{l}\text { Someros } \\
\text { con drenaje }\end{array}$ & $\begin{array}{l}\text { Buena } \\
\text { madera }\end{array}$ \\
\hline
\end{tabular}


1. Tiene una superficie o área de intersección o congruencia que abarca ligeramente más de la mitad del área de estudio (50,3\%), que era cercano al promedio $\left(1.795,2 \mathrm{~km}^{2}=48,1 \%\right)$ de los porcentajes de las áreas de intersección de las diferentes comunidades sintéticas (cuadro 2)

2. Tiene un número de especies superior al promedio (5) de las ocho especies estudiadas, por lo que podría ser también apropiada para otros fines tales como planes de manejo forestal o el enriquecimiento de plantaciones agrícolas, donde el número promedio de especies usadas es de dos (FAO 1992), y que ayuden en la reconversión de monocultivos a policultivos o se enfoquen hacia la agroforestería, al asociarse con otros cultivos (Somarriba et al. 2014).

3. Debido a que la mayoría de las especies escogidas son intolerantes a la sombra y pioneras en la sucesión ecológica, podrían establecerse todas las especies al mismo tiempo, lo que ayudaría en la regeneración natural de las zonas deforestadas de la región de estudio.

Por otro lado, la comunidad sintética de ocho especies también podría ser la idónea (figura 4B), en los siguientes aspectos:

1. Permitiría enriquecer la biodiversidad.

2. Ayudaría en el establecimiento de áreas dedicadas exclusivamente a la conservación, tales como la reserva ecológica estatal "Cañón del Usumacinta", lo que redundaría en un aumento en el ecoturismo y por lo tanto en los recursos económicos disponibles para la población local (Cámara et al. 2011).

3. Aumentarían los beneficios económicos a la población local al incluir la especie $C$. pentranda, ya que sus semillas pueden ser usadas para la producción de biocombustibles (Ong et al. 2013); mientras que la fibra de sus frutos se usan para el relleno de colchones, cojines y salvavidas (Sivakumar et al. 2013).

4. Podría contribuir en la disminución de los impactos de las inundaciones en la zona de estudio, como la ocurrida en el río Usumacinta durante 2008 que afectó la zona de estudio, al escoger esta comunidad sintética, ya que se ha descubierto que la mezcla de árboles pioneros con árboles tolerantes a la sombra, disminuyen la infiltración y aumentan la intercepción de la lluvia, lo que resulta en un menor escurrimiento de los ríos (Sprenger et al. 2013).

La formación de las comunidades sintéticas se debe de ajustar a las necesidades y objetivos de los tomadores de decisiones, en el sentido si lo que quieren es disponer de mayor área reforestada, pero con un reducido número de especies o si lo que se pretende es crear zonas con mayor biodiversidad, lo que redundará en un área menor donde puedan estar presentes más especies, pero intercaladas con aquellas especies con mayores áreas de distribución potencial. Los resultados obtenidos de la distribución potencial de las especies y la elaboración de las comunidades sintéticas son de gran utilidad para planear prácticas de conservación y de enriquecimiento del hábitat, que aumenten la biodiversidad, aunque con un número de especies limitado, haciendo que se gestionen de mejor manera los recursos destinados para tal fin, a la vez que se permite la inclusión de actividades económicas relacionadas con el aprovechamiento sustentable de dichas especies en proyectos a largo plazo, pero planificados desde el principio con planes de manejo forestal para especies, ya sea tropicales o templadas pero adaptados a una cierta zona de estudio.

Una de las principales limitaciones de este estudio es que está basado en distribuciones potenciales con probabilidades de ocurrencia de solo presencia. En investigaciones futuras, sería útil incluir también datos de ausencia. Se recomienda explorar cual va a ser el efecto del cambio climático en estas comunidades arbóreas en las próximas décadas. Otra posible limitación es el número de ocurrencias por especies, por lo que sería bueno consultar otras fuentes para tener mayor información. De igual manera, sería recomendable explorar comunidades sintéticas enfocadas hacia otros usos como biodiversidad o conservación.

\section{CONCLUSIONES}

Es posible diseñar comunidades sintéticas arbóreas que cumplan con los objetivos deseados por los tomadores de decisiones, solo que hay que tener muy en claro para lo que se quieren, porque si lo que se requiere es reforestar la mayor área posible de una cierta región, esto puede causar el detrimento del número de especies y por lo tanto de la biodiversidad, por lo que a veces hay que hacer un balance de los fines deseados, como aumentar la biodiversidad o el área reforestada, para encontrar la comunidad sintética más apropiada para una cierta región. En nuestro caso de estudio, donde el principal objetivo es la reforestación, se opta por abarcar la mayor área posible con un número de especies reducido. La principal aportación de este estudio es una nueva metodología para diseñar comunidades sintéticas arbóreas para el manejo de recursos forestales en zonas deforestadas, tanto en regiones tropicales como en extratropicales.

\section{AGRADECIMIENTOS}

Se agradece el apoyo brindado por parte del CONACYT por la beca de maestría por un año (Agosto 2014-Julio 2015) y del CCGSS mediante el proyecto TAB2012-C-28-194316 titulado "Retos para la sustentabilidad en la Cuenca del Río Usumacinta en Tabasco: ecosistemas, cambio climático y respuesta social" aprobado por el Fondo Mixto de Fomento a la Investigación Científica y Tec- 
nológica CONACYT- Gobierno del Estado de Tabasco, para la realización de los trabajos en campo, así mismo al posgrado de Ciencias Ambientales de la DACBiol-UJAT y a las personas que colaboraron en las salidas de campo a la zona de estudio. A mi papá (Eliazar) por su apoyo incondicional, al Dr. Arturo Valdés Manzanilla por su inmensa colaboración. Para recuerdo de mi extinto hermano (Jaco). A mi Dios por permitirme disfrutar de una mujer maravillosamente excepcional, llena de amor, bondad y ternura y poder cuidarla siempre y antes de fenecer, a la memoria de mi amada madre (la maestra Manuelita).

\section{REFERENCIAS}

Allouche O, A Tsoar, R Kadmon. 2006. Assessing the accuracy of species distribution models: prevalence, kappa and the true skill statistic (TSS). Journal of Applied Ecology 43: 1223-1232. DOI: https://doi.org/10.1111/j.13652664.2006.01214.x

Barros FSM, MF Siqueira, DP Costa. 2012. Modeling the potential geographic distribution of five species of Metzgeria Raddi in Brazil, aiming at their conservation. Bryologist 115:341-349.

Cámara CLC, TH Hernández, AO Castillo, AA Galindo, A Morales, LC Zequeira, FC Rullán, GMC Jesús, CLM Gama, GS Cappello, GM Ángeles. 2011. Estudio regional forestal de la UMAFOR de los Ríos. UJAT, 280 p. Consultado 15 sep. 2017. Disponible en http://www.conafor.gob.mx:8080/ documentos/docs $/ 9 / 3641$ Estudio $\% 20$ Regional $\% 20$ Forestal\%202709.pdf.

Caranqui J, D Suarez. 2012. Conservación y Estado Poblacional de Grosvenoria campii RM King \& H. Rob.(Asteraceae) en Ecuador. Q'EUÑA 5:17-24.

CONABIO (Comision Nacional para el Conocimiento y Uso de la Biodiversidad, MX). 2015. Red mundial de información sobre biodiversidad. Consultado 13 may. 2015. Disponible en http:/www.conabio.gob.mx/remib/doctos/remibnodosdb.html\#.

De Cauwer V, B Muys, R Revermann, A Trabucco. 2014. Potential, realised, future distribution and environmental suitability for Pterocarpus angolensis DC in southern Africa. $F_{O}$ rest Ecology and Management 315:211-226. DOI: https:// doi.org/10.1016/j.foreco.2013.12.032.

FAO (Food and Agriculture Organization, IT). 1992. Mixed and pure forest plantations in the tropics and subtropics. Rome, Italy. FAO forestry paper $103.151 \mathrm{p}$.

FAO (Food and Agriculture Organization, IT). 2016. State of the World's Forests 2016. Forests and agriculture: land-use challenges and opportunities. Rome, Italy. FAO. 107 p.

García-Aranda MA, C Cantú-Ayala, E Estrada-Castillón, M Pando-Moreno, A Moreno-Talamantes. 2012. Distribución actual y potencial de Taxus globosa (taxaceae) en México. Journal of the Botanical Research Institute of Texas 6:587-598.

GBIF (Global Biodiversity Information Facility). 2015. Search species occurrences. Consultado 10 feb. 2015. Disponible en: https://www.gbif.org/occurrence/search?q=

Griscom HP, MS Ashton. 2010. Restoration of dry tropical forests in Central America: a review of pattern and process. Forest Ecology and Management 261(10):1564-1579. DOI: https://doi.org/10.1016/j.foreco.2010.08.027.

Manjarrez MB, DS Hernández, B de Jong, TJ Nahed, VOO de Dios, ZEB Salvatierra. 2007. Configuración territorial y perspectivas de ordenamiento de la ganadería bovina en los municipios de Balancán y Tenosique, Tabasco. Investigaciones Geográficas 64:90-115.

Márquez IS, B de Jong, A Eastmond, GS Ochoa, S Hernández, JL Sandoval. 2008. Programas gubernamentales y respuestas campesinas en el uso del suelo: el caso de la zona oriente de Tabasco, México. Región y Sociedad 20(43):97-130.

Moreno SR, F Moreno-Sanchez, JM Torres-Rojo. 2011. National assessment of the evolution of forests fragmentation in Mexico. Journal of Forestry Research 23(4):529-541. DOI: https://doi.org/10.1007/s11676-011-0145-0.

Ong HC, AS Silitonga, HH Masjuki, TMI Mahlia, WT Chong, MH Boosroh. 2013. Production and comparative fuel properties of biodiesel from non-edible oils: Jatropha curcas, Sterculia foetida and Ceiba pentandra. Energy Conversion and Management 73:245-255. DOI: https://doi. org/10.1016/j.enconman.2013.04.011.

Pennington TD, J Sarukhán . 2005. Árboles Tropicales de México. Manual para la identificación de las principales especies. Universidad Nacional Autónoma de México. 523 p.

Phillips SJ, RP Anderson, RE Schapire. 2006. Maximum entropy modeling of species geographic distributions. Ecological Modelling 190(3):231-259. DOI: https://doi.org/10.1016/j. ecolmodel.2005.03.026

Sautu A, JM Baskin, CC Baskin, RS Condit. 2006. Studies on the seed biology of 100 native species of trees in a seasonal moist tropical forest, Panama, Central America. Forest Ecology and Management 234(1):245-263. DOI: https:// doi.org/10.1016/j.foreco.2006.07.006.

Sivakumar P, S Sindhanaiselvan, NN Gandhi, SS Devi, S Renganathan. 2013. Optimization and kinetic studies on biodiesel production from underutilized Ceiba Pentandra oil. Fuel 103:693-698. DOI: https://doi.org/10.1016/j. fuel.2012.06.029.

Somarriba E, A Suárez-Islas, W Calero-Borge, A Villota, C Castillo, S Vílchez, O Deheuvels, R Cerda. 2014. Cocoa-timber agroforestry systems: Theobroma cacao-Cordia alliodora in Central America. Agroforestry Systems 88(6):1001-1019. DOI: https://doi.org/10.1007/s10457-014-9692-7.

Sprenger M, Y Oelmann, L Weihermüller, S Wolf, W Wilcke, C Potvin. 2013. Tree species and diversity effects on soil water seepage in a tropical plantation. Forest Ecology and Management 309:76-86. DOI: https://doi.org/10.1016/j.foreco.2013.03.022.

Tudela F.1990. Recursos naturales y sociedad en el trópico húmedo tabasqueño. In Leff E ed. Medio Ambiente y Desarrollo en México Vol. I. Ciudad de México, México. Centro de Investigaciones Interdisciplinarias en Humanidades, UNAM. p. 149-189.

Tudela F. 1992. De la ilusión a la realidad: los grandes planes agroproductivos. In El Colegio de México-CINVESTAVIFIAS-UNRISD ed. La modernización forzada del trópico: el caso tabasqueño, Proyecto Integrado del Golfo. Ciudad de México, Mexico. p. 191-233.

UICN (Unión Internacional para la Conservación de la Naturaleza). 2017. The IUCN red list of threatened species. Consultado 15 sep. 2017. Disponible en http://www.iucnredlist.org/. Van Gils H, E Westinga, M Carafa, A Antonucci, G Ciaschetti. 
2014. Where the bears roam in Majella National Park, Italy. Journal of Nature Conservation 22:276-287. DOI: https:// doi.org/10.1016/i.jnc.2013.08.001

Vázquez NI, AO Castillo, HJI Valdez, CJ Zavala, SJL Martínez. 2011. Estructura y composición florística de la selva alta perennifolia en el ejido Niños Héroes Tenosique, Tabasco, México. Polibotánica 32:41-61.

Vessella F, B Schirone. 2013. Predicting potential distribution of Quercus suber in Italy based on ecological niche models: Conservation insights and reforestation involvements. Fo- rest Ecology and Management 304: 150-161. DOI: https:// doi.org/10.1016/j.foreco.2013.05.006.

World Bank 2017 Forest area (sq. km.). Consultado 10 ene. 2017. Disponible en: https://data.worldbank.org/indicator/ AG.LND.FRST.K2

Yang XQ, SPS Kushwaha, S Saran, JC Xu, PS Roy. 2013. Maxent modeling for predicting the potential distribution of medicinal plant. Justicia adhatoda L. in Lesser Himalayan foothills. Ecological Engineering 51:83-87. DOI: https:// doi.org/10.1016/J.ecoleng.2012.12.004

Recibido: 20/09/17

Aceptado: 25/03/19 
